\title{
Hyperbolic rules in genomes, the harmonic progression and recurrence sequences in algebraic biology
}

\author{
Sergey V. Petoukhov ${ }^{1}$ \\ ${ }^{1}$-Mechanical Engineering Research Institute of Russian Academy of Sciences. Russia, \\ 101990, Moscow, M. Kharitonievskiy pereulok, 4, \\ http://eng.imash.ru/, info@,imash.ru
}

\begin{abstract}
The paper is devoted to biological models using recurrence sequences, which are connected with the harmonic progression $1,1 / 2, \ldots, 1 / n$, and some cooperative properties of genomes. The harmonic progression is itself one of the recurrence sequences based on the harmonic mean. This progression appears in the hyperbolic rules of oligomer cooperative organization in eukaryotic and prokaryotic genomes. This allows thinking that the harmonic progression is also related to inherited physiological systems, which must be structurally consistent with the genetic coding system for their transmission to descendants and survival in evolution. The harmonic progression is one of historically known mathematical series, whose features were studied by Pythagoras, Leibniz, Newton, Euler, Fourier, Dirichlet, Riemann. It is widely used in many known algorithms and is closely related to some other important mathematical objects, for example, the function of the natural logarithm and harmonic numbers. Accordingly, the article describes the possibilities of using these interrelated mathematical objects to model biological structures, including logarithmic spirals and some other. Modeling inherited spiral configurations seems to be a particularly urgent task, since they are extremely common at all levels of organization of living bodies and, according to Goethe, are lines of life. The principle of a recurrence similarity, that is a special similarity of parts and transformations presented in recurrence sequences of numbers and matrix operators (the scale similarity and scale transformations are only particular cases of such similarity), is considered as one of the key principles of structural organization of living bodies.
\end{abstract}

Keywords: genomes, hyperbolic sequences, harmonic progression, recurrence sequence, harmonic numbers, natural logarithm, logarithmic spiral, biological spirals, tensor product.

\section{Introduction}

This article continues an analysis of the results of the author's study of the oligomer cooperative organization of eukaryotic and prokaryotic genomes. The first thematic results were described in [Petoukhov, 2020a,b] where a few hyperbolic rules of genomes were formulated, which are candidacies on the role of universal genomic rules. The descriptions of the rules used the following notions and materials, which are also used in this article and which we briefly recall.

Firstly, the oligomer sums method (or the OS-method) was introduced. The method relates to a consideration of each of nucleotide sequences in genomes as a sequence of DNA nucleotides A, T, C, and G (like as A-C-A-T-G-T-...), and also as a sequence of doublets (like as AC-AT-GT-GG-...), and as a sequence of triplets (like as ACA-TGT-GGA-...), etc. The method analyses numerically sets of $n$-plets (that is, oligomers with the length $n$ ), which belong the equivalence classes of $\mathrm{A}_{1}$-oligomers, or $\mathrm{T}_{1}$-oligomers, or $\mathrm{C}_{1}$-oligomers, or $\mathrm{G}_{1}$ - 
oligomers correspondingly (their index 1 indicates that all oligomers of each class start with the same nucleotide). For example, the class of the $A_{1}$-oligomers contains the following $n$ plets: 4 doublets AA, AT, AC, and AG; 16 triplets AAA, AAT, AAC, AAG, ATA, ..., AGG; etc.

Secondly, the application of this numeric method for studying different genomes has revealed the existence of a family of the universal hyperbolic rules for all analyzed eukaryotic and prokaryotic genomes. These rules concern the sequences of the total amounts $\Sigma_{\mathrm{A}, n, 1}, \Sigma_{\mathrm{T}, n, 1}, \Sigma_{\mathrm{C}, n, 1}$, and $\Sigma_{\mathrm{G}, n, 1}$ of different kinds of $n$-plets under $n=1,2,3, \ldots$ in each of the classes of $\mathrm{A}_{1^{-}}, \mathrm{T}_{1^{-}}, \mathrm{C}_{1^{-}}$, and $\mathrm{G}_{1}$-oligomers. These total amounts, regarding each of the classes, are members of the appropriate oligomer sums sequence (or briefly, an OS-sequence) of the class. It was discovered that these phenomenological OS-sequences are closely related with hyperbolic sequences $\mathrm{S}_{\mathrm{A}} / n, \mathrm{~S}_{\mathrm{T}} / n, \mathrm{~S}_{\mathrm{C}} / n$, and $\mathrm{S}_{\mathrm{G}} / n$, where $\mathrm{S}_{\mathrm{A}}, \mathrm{S}_{\mathrm{T}}, \mathrm{S}_{\mathrm{C}}$, and $\mathrm{S}_{\mathrm{G}}$ are correspondingly the numbers of nucleotides $\mathrm{A}, \mathrm{T}, \mathrm{C}$, and $\mathrm{G}$ in the considered nucleotide sequence (in this analysis, the author usually takes $n=1,2,3, \ldots, 20$ or, in special cases, $n=$ $1,2,3, \ldots, 99,100)$. For example, in the case of the human chromosome № 1 , a sequence of phenomenological values of the total amounts of $n$-plets from the class of $\mathrm{A}_{1}$-oligomers $[n$, $\left.\Sigma_{\mathrm{A}, n, 1}\right]$ lie along the hyperbolic sequence $\mathrm{S}_{\mathrm{A}} / n=67070277 / n$ with a high level of accuracy (the range of deviations is $-0.030 \% \div 0.024 \%$ ).

Similar situations hold for the OS-sequences of $n$-plets from classes of $\mathrm{A}_{1^{-}}, \mathrm{T}_{1^{-}}, \mathrm{C}_{1^{-}}$, and $\mathrm{G}_{1}$-oligomers in all human chromosomes and all other tested eukaryotic and prokaryotic genomes.

By appropriate scalings of numerators $\mathrm{S}_{\mathrm{A}}, \mathrm{S}_{\mathrm{T}}, \mathrm{S}_{\mathrm{C}}$, and $\mathrm{S}_{\mathrm{G}}$, each of the hyperbolic sequences $\mathrm{S}_{\mathrm{A}} / n, \mathrm{~S}_{\mathrm{T}} / n, \mathrm{~S}_{\mathrm{C}} / n$, and $\mathrm{S}_{\mathrm{G}} / n$ is transformed in the hyperbolic sequence (1):

$$
1 / n: \quad 1 / 1,1 / 2,1 / 3,1 / 4,1 / 5, \ldots, 1 / n
$$

The sequence (1) is known long ago as a harmonic progression where each term is the harmonic mean of the neighboring terms. For this reason, the revealed hyperbolic sequences in genomes allow speaking on the algebra-harmonic organization of genomes.

\section{Harmonic progression, harmonic mean and natural logarithms}

Let us consider a deep connection of the harmonic progression (1) with the harmonic mean, which in mathematics is one of several kinds of average, and in particular, one of the Pythagorean means. This connection is expressed in the known fact that the harmonic progression (1) is a recurrence sequence based on the harmonic mean relation between its members.

In general, the harmonic mean $H$ of the positive real numbers $x_{1}, x_{1}, \ldots, x_{n}$ is defined by the expression (2):

$$
H=\frac{n}{\frac{1}{x_{1}}+\frac{1}{x_{2}}+\cdots+\frac{1}{x_{n}}}=\frac{n}{\sum_{i=1}^{n} \frac{1}{x_{i}}}=\left(\frac{\sum_{i=1}^{n} x_{i}^{-1}}{n}\right)^{-1} .
$$

In accordance with the definition (2), if you have two numbers $x_{n}$ and $x_{n+2}$, then the harmonic mean $x_{n+1}$ between them is calculated by the relation (3):

$$
x_{n+1}=\left(\left(x_{n}^{-1}+x_{n+2}{ }^{-1}\right) / 2\right)^{-1}=2 x_{n} x_{n+2} /\left(x_{n}+x_{n+2}\right)
$$


Knowing two adjacent numbers $x_{n}$ and $x_{n+1}$ in this triplet of numbers and using the relation (3), the third number $x_{n+2}$ is determined through them by the recurrence equation (4):

$$
x_{n+2}=\mathrm{x}_{n} \mathrm{x}_{n+1} /\left(2 x_{n}-x_{n+1}\right)
$$

Repeated applications of the recurrence equation (4) generates a recurrence sequence $x_{n}$, $x_{n+1}, \ldots, x_{n+p}$. If $x_{1}=1$ and $x_{2}=1 / 2$, then this recurrence sequence is the harmonic progression (1): $1,1 / 2,1 / 3, \ldots, 1 / n$. So, the harmonic progression (1) is the recurrence sequence based on the harmonic mean relation (4).

The harmonic progression $1 / n(1)$ and hyperbola $y=1 / x$ have also deep relations with the function of the natural logarithm $\ln (x)$. The natural logarithm can be defined for any positive real number " $a$ " as the area under the hyperbola $\mathrm{y}=1 / x$ from 1 to $a$ (Fig. 1, at left) [Conway, Guy, 1995, p. 250]. In a polar coordinate system $[\theta, \mathrm{r}]$, the natural logarithm $\ln (x)$ - as the function of a variable $x$ - is represented by a spiral (see Fig. 1, at right; in this case, a variable $x$ plays the role of the angular coordinate $\theta$, and $\ln (x)$ plays the role of the radius-vector $\mathrm{r}$ ).
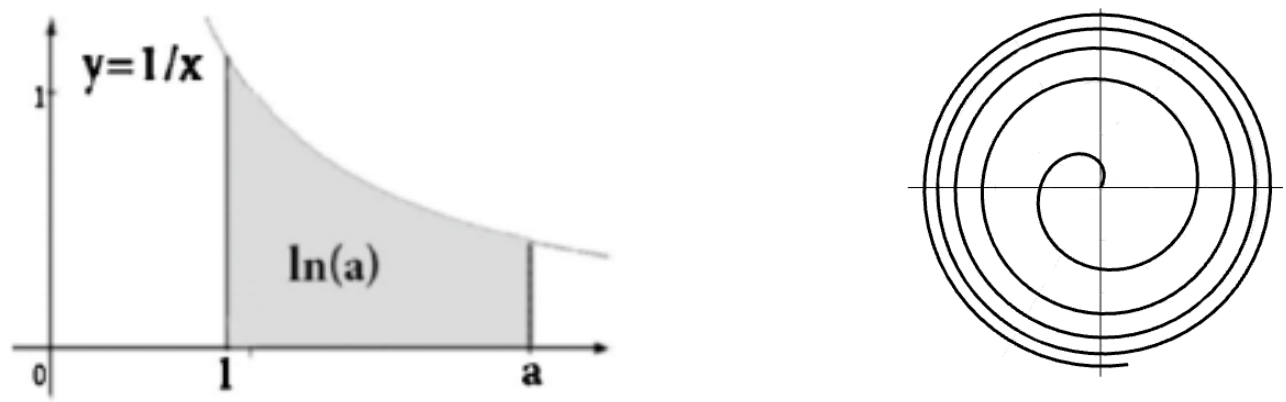

Fig. 1. At left: an illustration of the natural logarithm $\ln (x)$ as an area under the hyperbola $\mathrm{y}=1 / x$. At right: the function of the natural logarithm in the polar coordinate system.

The function of the natural logarithm is widely used in mathematical biology and relates to inherited biological phenomena. For example, different types of inherited sensory perceptions are subordinated to the main psychophysical law of Weber-Fechner: sight, hearing, smell, touch, taste, etc. The law states that the intensity of the perception is proportional to the logarithm of stimulus intensity; it is expressed by the equation (5):

$$
\mathrm{p}=\mathrm{k}^{*} \ln \left(x / x_{0}\right)=\mathrm{k}^{*}\left\{\ln (x)-\ln \left(x_{0}\right)\right\}
$$

where $\mathrm{p}$ - the intensity of perception, $\mathrm{x}$ - stimulus intensity, $\mathrm{x}_{0}$ - threshold stimulus, $\mathrm{ln}-$ natural logarithm, $\mathrm{k}-\mathrm{a}$ weight factor. Because of this law, for example, the power of sound in engineering technologies is measured on a logarithmic scale in decibels. The logarithmic nature of perception provides the body with a huge expansion of the range of perceived stimulus. For example, hammer strikes on a steel plate sound a hundred billion times louder than the quiet rustle of leaves, and the brightness of a voltaic arc trillions of times exceeds the brightness of some faint star, barely visible in the night sky [Vilenkin, 1985]. If sensations were proportional to stimuli, then the sensory organs would have an extremely difficult task to give sensations that differ billions and trillions of times. The organism with its genetically inherited sense organs bypasses this difficulty, as "logarithm" the irritations it receives. It is 
natural to think that this inherited ability of his is structurally linked with the genetic coding system, which ensures the coding of the sensory organs and their transmission to descendants. The described author's results give pieces of evidence on the connection of eukaryotic and prokaryotic genomes with the hyperbola $\mathrm{y}=1 / x$ and the corresponding natural logarithmic function.

Fig. 2 illustrates the connection of the hyperbola $y=1 / x$ with the logarithmic law of Weber-Fechner (5).

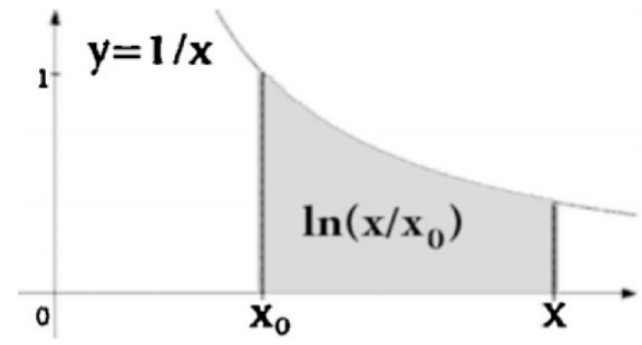

Fig. 2. An illustration of the connection of the hyperbola $y=1 / x$ with the logarithmic law of Weber-Fechner.

One can suppose that the innate Weber-Fechner law is the law, especially for the nervous system. But it is not so since its meaning is much wider because it holds true in many kinds of lower organisms without a nervous system in them: "this law is applicable to chemotropical, helio-tropical and geo-tropical movements of bacteria, fungi and antherozoids of ferns, ... . The Weber-Fechner law, therefore, is not the law of the nervous system and its centers, but the law of protoplasm in general and its ability to respond to stimuli" [Shults, 1916].

Taking into account the connection of the function of the natural logarithm with the hyperbola $y=1 / x$, one can note that the recurrence harmonic sequence $1 / n(1)$ is related with another recurrence sequence of the natural logarithms $\ln (n)(6)$ :

$$
\ln (1), \ln (2), \ln (3), \ldots, \ln (n)
$$

Fig. 3 shows this recurrence sequence (6) in a graphical form in the polar coordinate system, where a variable $n$ plays the role of the angular coordinate $\theta$, and $\ln (n)$ plays the role of the radius-vector $r$.

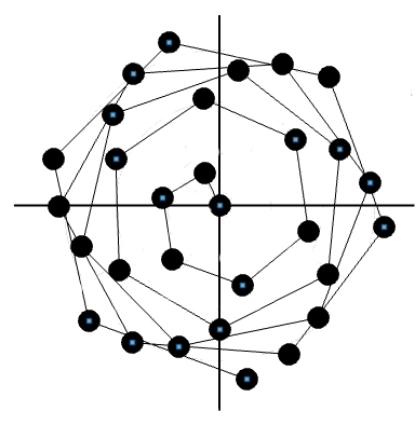

Fig. 3. A graphical representation of the function of the natural logarithm $\ln (n)$ in the polar coordinate system at $n=1,2, \ldots, 30$.

The natural logarithm function $\mathrm{y}=\ln (x)$, which is important to model inherited biological phenomena, is mutually inverse to the exponent function $\mathrm{x}=\exp (y)$, which is also used long ago to model inherited biological phenomena including morphogenetic phenomena. 
Correspondingly the recurrence logarithmic sequence (6) is mutually inverse to the recurrence exponential sequence $\exp (n)$ :

$$
\exp (1), \exp (2), \exp (3), \ldots, \exp (n)
$$

Fig. 4 shows graphical representations of the continuous exponential function exp (x) and discrete recurrence exponential sequence (7) in the polar coordinate system. These graphs represent continuous and discrete logarithmic spirals.
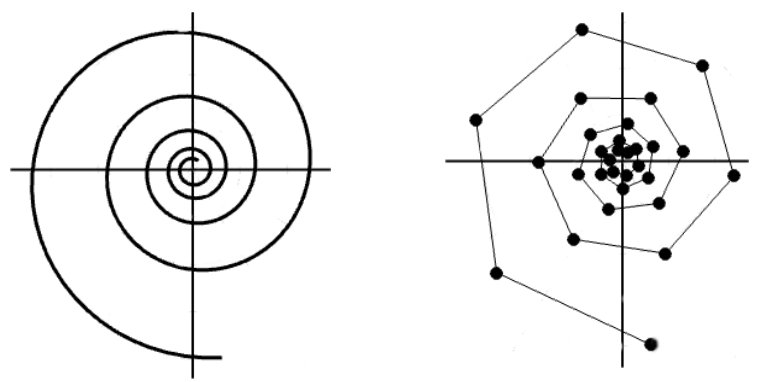

Fig. 4. Graphical representations of the continuous exponential function exp $(0.1 x)$ and the discrete recurrence sequences $\exp (0.1 n)$ at $n=1,2, \ldots, 30$.

Logarithmic functions and logarithmic spirals are realized in many inherited biological phenomena including the phyllotaxis patterns of morphogenesis [Jean, 1994], which are related to another recurrent sequence - the sequence of Fibonacci numbers. Fig. 5 shows a few examples of logarithmic spirals in inherited morphological structures.
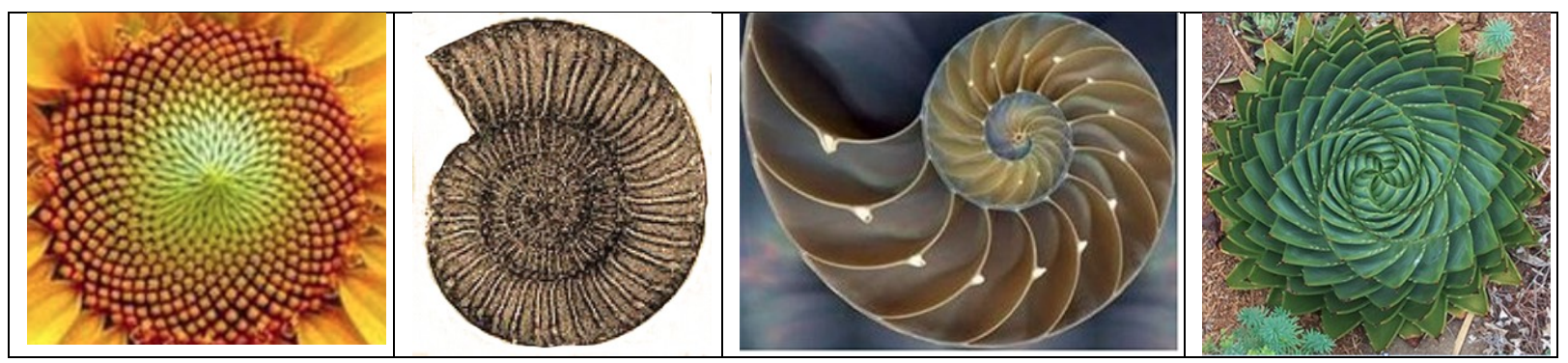

Fig. 5. Examples of logarithmic spirals in morphogenetic configurations: sunflower head, two clamshells, and aloe.

Regarding a question on a biological interrelation between the natural logarithm function $\ln (x)$ and the exponentiation function exp $(x)$, one can note that sensory organs, whose functions correspond the logarithmic law of Weber-Fechner, have in their morphology forms that are close to being many logarithmic spirals, which correspond the exponential function, for example, in the organs of hearing and vision:

- The human cochlea is traditionally compared to the shell of the nautilus, which is a near-perfect example of a logarithmic spiral. Besides this, the organ of hearing has spiral ganglia, spiral grooves, a spiral limb, etc.;

- In the organ of vision, corneal nerves of the subepithelial layer terminate near the superficial epithelial layer of the cornea in a logarithmic spiral pattern [Yu, Rosenblatt, 2007].

It can be added that some inherited instincts and automatisms of behavior in animals are also associated with the logarithmic spirals, for example: 
- The widespread garden spider Epeira weaves its web in the form of a logarithmic spiral [Gazale, 1999, p. 3; Hammer, 2016]. Fig. 6, at left and middle, shows such web and its model in a form of a discrete logarithmic spiral, which is drawn in Matlab by the following command: $\mathrm{n}=0$ :pi/10:11* $\left.\mathrm{pi} ; \mathrm{r}=130^{*}\left(2 .^{\wedge}(1 / 12)\right)\right)^{\wedge} n$; $\operatorname{polar}(\mathrm{n}, \mathrm{r})$;

- Birds of prey circle over their prey in a logarithmic spiral, and so on [Chin, 2000; Himmelman, 2002].

In music, the sound frequencies $\mathrm{f}$ of notes in an equal-tempered scale is expressed regarding a basic frequency $\mathrm{f}_{0}$ by the recurrent relation $\mathrm{f}=\mathrm{f}_{0} *\left(2^{1 / 12}\right)^{n}$; a corresponding recurrent sequence of frequencies is represented in the polar coordinate system by a discrete logarithmic spiral in Fig. 6, at right. This graph is built in Matlab by the following command: $n=0: 2 \wedge^{\wedge}(1 / 12): 73 ; \mathrm{r}=130^{*}\left(2 .^{\wedge}(1 / 12)\right) .^{\wedge} n ; \operatorname{polar}(n, \mathrm{r})$. Here $\mathrm{f}_{0}=130 \mathrm{~Hz}, n=1,2, \ldots, 73$.

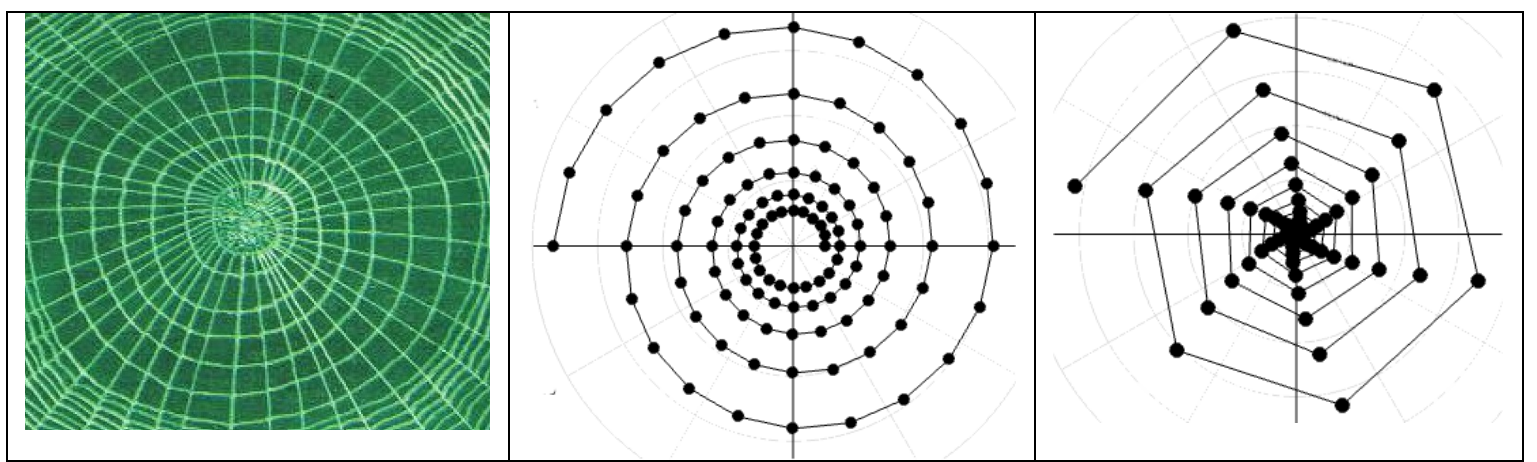

Fig. 6. Some biological examples of discrete logarithmic spirals. Left: a web of the garden spider Epeira. Middle: a graphical representation of a model of this web model as a recurrent sequence in polar coordinates. Right: a recurrence sequence of note frequencies in 6 octaves of the equal-tempered scale in the polar coordinates.

Recurrence sequences are very appropriate for biological modeling since they have a discrete nature, which corresponds discrete nature of biological systems: the genetic code system, the nervous system with its information impulses in neurons, sensory perception systems (differential thresholds of sensory perception exist), etc.

The recurrence harmonic sequence (1) determines another interesting recurrence sequence known long ago, which is considered in the next section.

\section{Harmonic progression and harmonic numbers}

The amounts of the initial members of the harmonic progression (1) are termed harmonic numbers $\mathrm{H}_{n}$ [Graham, Knuth, Parashnik,1994; Sondow, Weisstein 2020]. These numbers appear so often in the analysis of algorithms that computer scientists need a special notation for them [Graham, Knuth, Parashnik,1994, p. 273]. The series (8) presents first harmonic numbers $\mathrm{H}_{n}$ :

$$
\mathrm{H}_{n}=\sum_{k=1}^{n} 1 / k: 1,3 / 2,11 / 6,25 / 12,137 / 60, \ldots
$$

Harmonic numbers are related to the harmonic mean in that the $n$-th harmonic number is also $n$ times the reciprocal of the harmonic mean of the first $n$ positive integers $1,2,3, \ldots, n$.

Those hyperbolic rules of oligomer cooperative organization of genomes, which were formulated with respect to hyperbolic sequences in [Petoukhov, 2020], can be reformulated by using the notion of harmonic numbers $\mathrm{H}_{n}$. For example, the first hyperbolic rule dealing individual chromosomes can be reformulated in the following manner: 
- For any of classes of $\mathrm{A}_{1^{-}}, \mathrm{T}_{1^{-}}, \mathrm{C}_{1^{-}}$, or $\mathrm{G}_{1^{-}}$-oligomers, the sum of the first members of its oligomer sums sequence $\Sigma_{\mathrm{N}, n, 1}(n)$ is equal to an appropriate harmonic number $\mathrm{H}_{n}$ multiplied by the number $\mathrm{S}_{\mathrm{N}}$ of appropriate nucleotides $\mathrm{N}$, that is, $\mathrm{H}_{n}{ }^{*} \mathrm{~S}_{\mathrm{N}}$ (here $\mathrm{N}$ denotes any of nucleotides $\mathrm{A}, \mathrm{T}, \mathrm{C}$, or $\mathrm{G} ; \mathrm{S}_{\mathrm{N}}$ denotes an amount of the nucleotide $\mathrm{N} ; n$ $=1,2,3,4, \ldots)$.

Harmonic numbers are connected with the function of natural logarithm: the harmonic number $\mathrm{H}_{n}$ is the discrete analog of the continuous function $\ln (\mathrm{x})$ [Graham, Knuth, Parashnik, 1994, p. 276]. Fig. 7 shows an approximation of the function of the natural logarithm in Fig. 2 by the sum of areas $1,1 / 2,1 / 3,1 / 4, \ldots, 1 / n$ of the shown rectangles; sums of these areas form the series of harmonic numbers $\mathrm{H}_{n}(1)$.

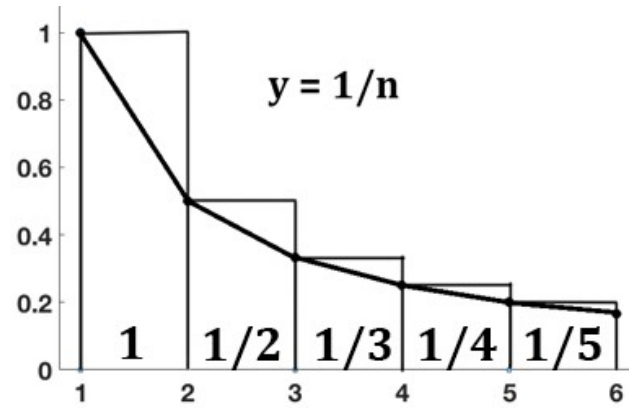

Fig. 7. An approximation of the area under the hyperbola $y=1 / x$ by the sum of areas $1,1 / 2,1 / 3,1 / 4, \ldots, 1 / n$ of the shown rectangles, whose areas form the series of harmonic numbers $\mathrm{H}_{n}(8)$.

In 1740, Euler obtained an asymptotic expression for the harmonic numbers $\mathrm{H}_{n}$ :

$$
\mathrm{H}_{n}=\ln (n)+\gamma+\varepsilon
$$

where $\gamma=0.5772 \ldots$ is the Euler-Mascheroni constant, and the value $\varepsilon$ tends to zero with increasing $n$. Therefore, with increasing $n$, the harmonic number $\mathrm{H}_{n}$ is more and more accurately expressed by the sum (10):

$$
\mathrm{H}_{n} \approx \ln (n)+0.5772=\ln (n)+\ln (1.7810)=\ln \left(1.7810^{*} n\right)
$$

So, the sequence of harmonic numbers $\mathrm{H}_{n}$ (8) is graphically represented in the polar coordinate system $(\mathrm{r}, \theta)$ by a discrete spiral curve in Fig. 8; in this case $\mathrm{r}=\mathrm{H}_{n}$ and $\theta=n$.

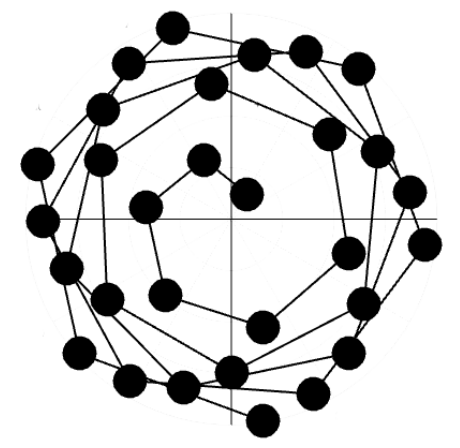

Fig. 8. The graphic representation of the series of harmonic numbers $H_{n}(8)$ in the polar coordinate systems under $n=1,2,3 \ldots, 30$. This discrete spiral configuration is one of 
the representations of universal properties of eukaryotic and prokaryotic genomes formulated in the hyperbolic rules of their oligomer cooperative organization [Petoukhov, 2020a,b].

One should note that if you consider a hyperbolic sequence like $\mathrm{S} / n$, where $\mathrm{S}$ denotes the number of a certain kind of nucleotides, the sum of its first members is equal to an appropriate harmonic number $\mathrm{H}_{n}$ multiplied by the scale factor $\mathrm{S}$ (11):

$$
\sum_{k=1}^{n} \mathrm{~S} / k=\mathrm{S}^{*} \sum_{k=1}^{n} 1 / k
$$

Therefore, the corresponding graph of the genomic sequence $\sum_{k=1}^{n} \mathrm{~S} / k$ in polar coordinates is represented by the same discrete spiral as in Fig. 8 but only on another scale.

To improve an approximation of the logarithmic function $\ln (x)$ by the total area of the rectangles, you can consider - instead of the classic harmonic progression $1,2, \ldots, 1 / k$ - more fractional sequences, called the "compressed harmonic progression" (or briefly, the C-harmonic progression):

$$
1,1+1 / m, 1+2 / m, \ldots, 1+k / m,
$$

where the additional factor $m$ can take integer values $1,2,3, \ldots$. The amounts of the initial members of the C-harmonic progression (12) are termed by author as "compressed harmonic numbers" (or C-harmonic numbers). Fig. 9, at right, shows an improved approximation of the continuous function $\ln (x)$ by C-harmonic numbers $\mathrm{H}_{n}{ }^{[m]}$ at $m=3$ : the area under the hyperbola, representing the natural logarithm, is better approximated by the total area of the rectangles equal to the $\mathrm{C}$-harmonic number $\mathrm{H}_{n}{ }^{[3]}$. The larger the value $m$, the better the approximation of the continuous function $\ln (x)$ by discrete C-harmonic numbers $\mathrm{H}_{n}{ }^{[m]}$ is provided.

Replacing $\ln (x)$ and $\ln \left(x_{0}\right)$ in the expression (5) of the Weber-Fechner law by corresponding C-harmonic numbers $\mathrm{H}_{n}{ }^{[m]}$ and $\mathrm{H}_{r}^{[m]}$, where integer $\mathrm{r}<\mathrm{n}$, we get the equation (13):

$$
p=v^{*}\left\{\sum_{k=0}^{m(n-1)}(1+k / m)-\sum_{k=0}^{r}(1+k / m)\right\}=v^{*} \sum_{k=r+1 / m}^{m(n-1)}(1+k / m)
$$

The equation (13) is the discrete analog of the Weber-Fechner law based on the harmonic progression and $\mathrm{C}$-harmonic numbers.

The author believes that harmonic numbers $\mathrm{H}_{n}$ and compressed harmonic numbers $\mathrm{H}_{n}{ }^{[m]}$ will be useful for modeling inherited physiological systems and processes by recurrence sequences, including the modeling biological spiral systems and processes in their relation to the genetic coding system. 

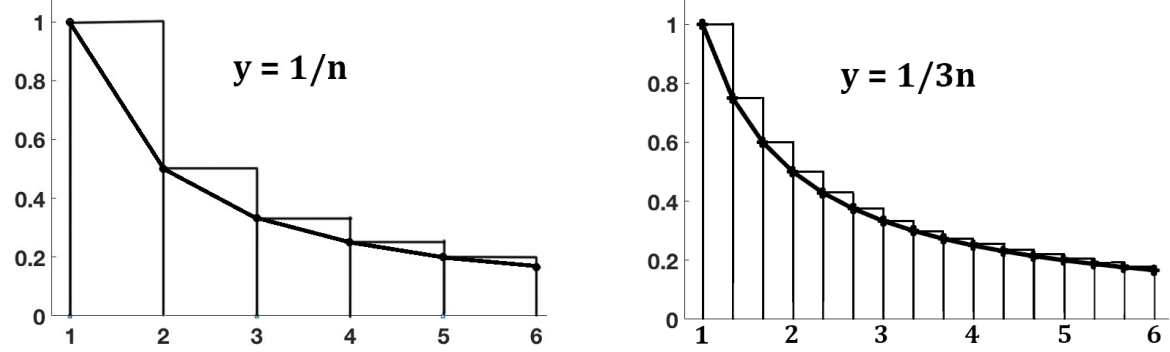

Fig. 9. A comparison of the approximations of continuous function $\ln (x)$, presented in a Cartesian coordinate system, by classical harmonic numbers $\mathrm{H}_{n}$ (at left) and by compressed harmonic numbers $\mathrm{H}_{n}{ }^{[m]}$ under $m=3$ (at right).

As is known, the spiral organization of living bodies and processes in them is one of the fundamental features of living matter. Goethe even called spirals "symbols of life" because of multiple implementations of inherited spiral structures and processes in living bodies at various lines and levels of biological evolution. In the human body spiral structures, genetically inherited from one generation to another, exist in the muscles, heart, blood vessels, bones, nerves, the organ of hearing (the cochlea), cellular organization of the embryo (zygote), etc. [Petoukhov, Svirin, Khazina, 2015]. The structure of tendons and ligaments consists of spirals, which in turn are composed of collagen that has a triple spiral structure. Spiral motions (nutation) are observed during the growth of roots and shoots, tendrils of plants are spirally wrapped, a tissue in the trunks of trees grows spirally, etc. Because of spiral configurations, all the fluids in the body (blood, lymph, and urine) are spiral. The title of a book about bio-spirals, "Lines of Life" [Cook, 1914] reflects their importance for living matter.

Spirals in living matter are quite different from spirals in inanimate nature:

1. They are genetically inherited in the chain of generations and are associated with structures of a molecular-genetic system that is full of spirals and super spirals at different levels of its organization, from DNA to chromosomes;

2. Characteristics of biological spirals are associated with Fibonacci numbers at different levels and branches of the biological evolution, from alpha-peptides to the structure of the integral bodies of plants and animals, which is reflected in the famous biomathematical laws of phyllotaxis, to which thousands of publications have been devoted worldwide [Jean, 1994].

\section{Recurrence sequences of genetic matrices}

Above we talked about recurrent sequences of numbers. But the notion of recurrence sequences of numbers can be generalized to a notion of recurrence sequences of matrices including tensor recurrence sequences of matrices. The author's studies of the systems of the alphabets of the molecular genetic system showed that their structural features are associated with tensor recurrence sequences of matrices, for example, $[\mathrm{C}, \mathrm{A} ; \mathrm{T}, \mathrm{G}]^{n}$, or $[\mathrm{G}, \mathrm{T} ; \mathrm{C}, \mathrm{A}]^{(n)}$ where $n=1,2,3, \ldots$ The systematic analysis of these tensor recurrence sequences, proposed and carried out by the author since 2008 , has led to many meaningful results about the hidden relationship of genetic and physiological subsystems, as described in many publications, for example, [Petoukhov, 2008, 2011, 2012, 2016, 2017-2019, 2020a; Petoukhov, He, 2010; 
Petoukhov, Petukhova, 2017; Petoukhov, Petukhova, Sirin, 2019; Petoukhov, Tolokonnikov, 2020].

In particular, the hyperbolic rules of oligomer cooperative organization of eukaryotic and prokaryotic genomes [Petoukhov, 2020a], connected with the harmonic progression (1), were revealed based on analysis of such tensor recurrence sequences of genetic matrices. Similtaneously tensor recurrence sequences of matrices have also proven useful for analyzing and modeling the Mendel's laws of inheritance of traits, phonetic sequences in long Russian literary texts [Petoukhov, 2011, 2020a] and some other bio-phenomena. The study of such tensor recurrence sequences in genetics has led to the quantum-informational model of long DNA sequences [Petouhkov, 2018; Petoukhov, Petukhova, Svirin, 2019]. Tensor recurrence sequences of genetic matrices draw attention to an idea that the principle of recurrence sequences is essential not only for morphological configurations but also for sets of matrix operators representing transformations and cooperations of separate parts in living bodies.

The author believes that structural organization of living bodies is based - in an essential degree - on recurrence sequences of matrices including tensor recurrence sequences of matrices. In other words, the principle of a recurrence similarity, that is a special similarity of parts and transformations presented in recurrence sequences of numbers and matrix operators (the scale similarity and scale transformations are only particular cases of such similarity), seems to be one of key principles of structural organization of living bodies. Briefly speaking, living bodies are constructed based on recurrences in a great degree.

\section{Some concluding remarks}

The article shows the usefulness of recurrent sequences, which are associated with the structural features of the molecular genetic system, for modeling genetically inherited biological structures including spiral configurations as the very important class of structures. Living matter appears as an algebraic-harmonic entity. One can separately note the result on that the cooperative system of oligomers in the genomes are associated with the harmonic progression, which is widely known in connection with musical harmony and the frequency system of musical overtones. But the harmonic progression is important not only in music. At least from the time of the Pythagorean doctrine of the aesthetics of proportions, the following idea exists: "the aesthetic principle is the same in every art; only the material differs" [Schumann, 1969]. In light of this, architecture has long been interpreted as frozen music, and music as dynamic architecture. Additional speculation about the possible genetic basis of some aesthetic parallels in various arts arises though the very idea of the connection between the feeling of beauty and the genetic system is not new: it is reflected, for example, in the title of the article "Beauty is in the genes of the beholder" about a connection of some parameters of the DNA double helix with the golden section [Harel, Unger, Sussman, 1986].

Recurrent sequences determine much in the configurations of living bodies, whose morphological mysteries of the formation have long been of interest to people. D'Arcy W. Thompson in his famous book "On Growth and Form" (1917) discussed the intricate and complex forms that can emerge in biology. He emphasized that the analytical approach to biological form can provide answers on the forces that acted during its (ontogenetic or phylogenetic) development. In our dates, mathematical models of morphological configurations can be used in reconstructing the shape of individual organs based on the limited individual information from clinical computer tomography by matching it with the 
analytical model of the shape. The author believes that the application of the theory of multiagent tensor recurrent sequences and functions to the study of biological forms is, in particular, an adequate way of understanding the families of those two-dimensional and three-dimensional biological spirals that are "the curves of life" [Cook, 1914]. On this path is the knowledge of those unified algorithms of biological structuring that operate on different lines and branches of biological evolution and the result of which was expressed by Goethe in the phrase "Everything is a leaf" [Conway, Guy, 1995].

Regarding these unified biological algorithms, special attention should be paid to the study of the phenomena of inheritance in animals of the ability to actions of an intellectual nature. For example, the garden spider Epeira weaves its web in the form of the logarithmic spiral (Fig. 6) on the basis of its inherited instincts [Hammer, 2016]. This job requires a certain amount of intelligence as the spider attaches the ends of the web to random supports and uses different types of filaments. Therefore, one can believe that intellectual activity is associated with algorithms of morphogenesis and is also associated with recurrence sequences and harmonic numbers, which are a discrete analog of the logarithmic function. This supports the following thoughts on artificial intelligence: "artificial intelligence could be brought closer to mathematical thinking if it were possible to realize the metrical properties of the human mind. ... the consciousness itself is structured geometrically: any person in his existential aspects is geometric. ... in our minds when constructing texts through which we perceive the World, something very similar to what happens in morphogenesis occurs. We are ready to see in the depths of consciousness the same geometric images that are revealed in morphogenesis" [Nalimov, 2015, p. 115].

During the research described in this article, the interaction between neighboring molecules, for example, according to the well-known principle "key-and-lock" was not used at all, and nevertheless, meaningful results were obtained. These results show the existence of cooperative forms of behavior of biological molecules when many molecules behave as a whole (similar to the cooperative behavior of physical elements in lasers). The body of an adult contains about 100 trillion cells, which are combined into a whole organism with cooperative forms of behavior. The study of bio-symmetries and biological recurrence sequences makes it possible to identify and study these collective forms of behavior of astronomical quantities of biomolecules.

This relates to many theoretical and technological tasks. Corresponding problems are discussed at the International interdisciplinary seminar "Algebraic Biology and Theory of Systems" in Moscow [Petoukhov, Tolokonnikov, 2020].

\section{Acknowledgments}

Some results of this paper have been possible due to long-term cooperation between Russian and Hungarian Academies of Sciences on the theme "Non-linear models and symmetrologic analysis in biomechanics, bioinformatics, and the theory of self-organizing systems", where the author was a scientific chief from the Russian Academy of Sciences. The author is grateful G. Darvas, E. Fimmel, M. He, Z.B. Hu, Yu.I. Manin, I.V. Stepanyan, V.I. Svirin and G.K. Tolokonnikov for their supports. The author is also grateful the members of the International Symmetry Association (Budapest, http://isa.symmetry.hu/) and the International Seminar "Algebraic Biology and System Theory" (Moscow, 
https://www.youtube.com/channel/UC8JLsuRzzPsRiHwrwEjMCtw) for discussing the author's researches in the field of matrix genetics and algebraic biology.

\section{References}

Chin G.J. (2000). Organismal Biology: Flying Along a Logarithmic Spiral. Science, vol. 290 (issue 5498): pp.1857, doi:10.1126/science.290.5498.1857c https://science.sciencemag.org/content/290/5498/1857.3

Conway J.H., Guy R.K. (1995). The Book of Number. N.-Y.: Copernicus, ISBN 0-38797993-X.

Cook T.A. (1914). The Curves of Life. London, Constable and Co, $490 \mathrm{p}$.

D'Arcy Wentworth Thompson (1992). On Growth and Form: The Complete Revised Edition. Dover Publications. ISBN-10: 0486671356, ISBN-13: 978-0486671352

Fimmel E., Petoukhov S.V. (2020) Development of Models of Quantum Biology Based on the Tensor Product of Matrices. In: Hu Z., Petoukhov S., He M. (eds) Advances in Artificial Systems for Medicine and Education III. AIMEE 2019. Advances in Intelligent Systems and Computing, vol 1126, p.126-135. Springer, Cham DOI https://doi.org/10.1007/978-3-030-39162-1_12

Gazale M.J. (1999). Gnomon. From Pharaons to Fractals. Princeton Univ. Press. ISBN 0691-00514-1.

Graham R.L., Knuth D.E., Patashnik O. (1994). Concrete mathematics : a foundation for computer science. 2nd ed., ISBN 0-201-55802-5.

Hammer Ø. (2016) The Spider's Spiral Spin. In: The Perfect Shape. Copernicus, Cham. https://doi.org/10.1007/978-3-319-47373-4_52

Harel D., Unger R., Sussman J.L. (1986). Beauty is in the genes of the beholder. Trends in Bioch. Sc., 11, p. 155-156.

Himmelman J. (2002). Discovering Moths: Nighttime Jewels in Your Own Backyard. Down East Enterprise Inc., p. 63. ISBN 978-0-89272-528-1.

Jean R. (1994). Phyllotaxis. A systemic study in plant morphogenesis. Cambr. Univ. Press.

Nalimov V.V. (2015). I am scattering thoughts (in Russian: Razbrasyvaiu mysli). - Center for Humanitarian Initiatives; Moscow ISBN 978-5-98712-521-2.

Petoukhov S.V. (2008). Matrix genetics, algebrases of genetic code, noise immunity. Moscow, RCD, 316 p. (in Russian).

Petoukhov S.V. (2011). Matrix genetics and algebraic properties of the multi-level system of genetic alphabets. Neuroquantology, v. 9, №4, p. 60-81.

Petoukhov S.V. (2012). Symmetries of the genetic code, hypercomplex numbers and genetic matrices with internal complementarities. Symmetry: Culture and Science, vol. 23, № 3-4, p. 275-301.

Petoukhov S.V. (January 2016). The system-resonance approach in modeling genetic structures. Biosystems, v. 139, p. 1-11.

Petoukhov S.V. (2017). Genetic coding and united-hypercomplex systems in the models of algebraic biology. Biosystems, v. 158, August 2017, pp. 31-46.

Petoukhov S.V. (2018a). The Genetic Coding System and Unitary Matrices. Preprints 2018, 2018040131, doi: 10.20944/preprints201804.0131.v2.

http://www.preprints.org/manuscript/201804.0131/v2

Petoukhov S.V. (2018b). The rules of long DNA-sequences and tetra-groups of oligonucleotides. arXiv:1709.04943v5, 5th version from 8 October 2018, 159 pages (2018b). 
Petoukhov S.V. (2019). Connections Between Long Genetic and Literary Texts. The Quantum-Algorithmic Modelling. In: Hu Z., Petoukhov S., Dychka I., He M. (eds) Advances in Computer Science for Engineering and Education II. pp 534543, ICCSEEA 2019. Advances in Intelligent Systems and Computing, v. 938. Springer, Cham. Online publication on 29 March 2019. (2019b). https://link.springer.com/chapter/10.1007/978-3-030-16621-2_50\#citeas .

Petoukhov S.V. (2020a). Hyperbolic Rules of the Oligomer Cooperative Organization of Eukaryotic and Prokaryotic Genomes. Preprints 2020, 2020050471, 95 pages. doi: 10.20944/preprints202005.0471.v2, https://www.preprints.org/manuscript/202005.0471/v2

Petoukhov S.V. (2020b). Genomes symmetries and algebraic harmony in living bodies. Symmetry: Culture and Science, v. 31, No. 2, p. 222-223, https://doi.org/10.26830/symmetry_2020_2_222.

Petoukhov S.V., He M. Symmetrical Analysis Techniques for Genetic Systems and Bioinformatics: Advanced Patterns and Applications. IGI Global, USA (2010). http://petoukhov.com/Petoukhov, $\% 20 \mathrm{He} \% 20-\% 202010 \% 20-$

\%20Symmetrical $\% 20$ Analysis $\% 20$ Techniques $\% 20$ for $\% 20$ Genetic $\% 20$ Systems $\% 20$ and $\% 20$ Bioinformatics.pdf.

Petoukhov S.V., Petukhova E.S. Symmetries in genetic systems and the concept of genological coding. - Information, 2017, 8(1), 2 (2017a). doi:10.3390/info8010002, http://www.mdpi.com/2078-2489/8/1/2/htm

Petoukhov S.V., Petukhova E.S., Svirin V.I. Symmetries of DNA alphabets and quantum informational formalisms. Symmetry: Culture and Science, v. 30, No. 2, pp. 161-179 (2019), https://doi.org/10.26830/symmetry_2019_2_161,

http://petoukhov.com/PETOUKHOV\%20GENETIC\%20QUANTUM\%20INFORMATIONA L\%20MODEL\%202019.pdf

Petoukhov S.V., Tolokonnikov S.V. (2020) Algebraic biology and matrix genetics systems. - Presentation at the International interdisciplinary seminar "Algebraic Biology and Theory of Systems" 02/13/2020, Moscow, Russia (in Russian), https://www.youtube.com/watch?v=H2dNtvTM11M\&t=330s.

Shults E. (1916). The organism as creativity. In the book "Questions of theory and psychology of creativity" ("Voprosy teorii i psikhologii tvorchestva"), Russia, Kharkov, vol.7, pp. 108-190 (1916) (in Russian).

Schumann R. (1969). On Music and Musicians, ed. Konrad Wolff. New York.

Sondow J., Weisstein E. W. (2020). Harmonic number. From MathWorld - A Wolfram Web Resourse, https://mathworld.wolfram.com/HarmonicNumber.html

Vilenkin N.Ya. (1985). Functions in nature and technology (Funktsii v prirode i tehnike). Moscow: Prosveschenie, 1985 (in Russian).

Yu C.Q., Rosenblatt M.I. (2007). Transgenic corneal neurofluorescence in mice: a new model for in vivo investigation of nerve structure and regeneration. Invest Ophthalmol Vis Sci. ; 48(4): pp. 1535-42 